\title{
Applying the Combined Phacoemulsification and Trabeculectomy to Treat Cataract Combined with Glaucoma X He${ }^{1}$, X Chen ${ }^{2}$, X Chen ${ }^{1}$, B Ning ${ }^{1}$, H Yin $^{1}$
}

\begin{abstract}
Objective: It is a common disease in clinic treatment and the vision of patients cannot be effectively improved simply by the glaucoma surgery.

Methods: By retrospectively analyzing the clinical data of treating 40 cases (48 eyes) of cataract combined with glaucoma by the combined phacoemulsification and trabeculectomy, this paper is to discuss the clinical effect on cataract combined with glaucoma treatment by applying the combined phacoemulsification and trabeculectomy.
\end{abstract}

Results: After the treatment of patients, their intraocular pressure and anterior chamber depth were well recovered, the vision was significantly improved and the postoperative morbidity was only $10.42 \%$.

Conclusion: It can be seen that the combined phacoemulsification and trabeculectomy is an effective way to treat the cataract combined with glaucoma with a more ideal therapeutic effect.

Keywords: Phacoemulsification, trabeculectomy, cataract, glaucoma

From: ${ }^{1}$ Karamay Central Hospital of Xinjiang, Karamay, 834000, China, ${ }^{2}$ The First Affiliated Hospital of Xinjiang Medical University, Wulumuqi, 830054, China.

Correspondence: Dr X He, Karamay Central Hospital of Xinjiang, Karamay, 834000

China.Email: pxys006@126.com 


\section{INTRODUCTION}

The cataract is a common disease in clinic treatment, the vision of patients cannot be effectively improved simply by the glaucoma surgery. Not only that, it would aggravate the visual dysfunction and seriously impact the therapeutic dependence (1). Applying the trabeculectomy can promote the continuous developed phacoscotasmus and accelerate the speed of formed cataract, for which case the patients should undergo a second surgery (2). As medical technologies continuously develop, the combined phacoemulsification and trabeculectomy has been widely applied to the clinic treatment of cataract combined with glaucoma (3). This means for cataract combined with glaucoma can gain a more significant improvement and present a more significant efficacy.

\section{SUBJECTS AND METHOD}

The combined phacoemulsification and trabeculectomy is applied to treat cataract combined with glaucoma. The surgery of cataract combined with glaucoma can be seen in Figure 1. The regular slit lamp, A/ B ultrasonic for eyes, light projection and chromoptometry need to be examined before the surgery. The lens under a state of glaucoma is presented in Figure 2.

This paper calculated the diopter of required intraocular lens and carried out conventional full-body medical examination for patients. Before the surgery, patients were given the related drugs for the effective reduction of intra-ocular pressure along with diclofenac sodium eyedrops and for those patients with serious conjunctival congestion, the corticosteroid eyedrops were given for relief. The intravenous dripped mannitol (200g/L) was given half hour before and the compound tropicamide eyedrops were given for mydriasis 15 
minutes before the surgery. The 1:200 of epinephrine was injected in anterior chambers for patients with non-ideal effects of mydriasis. The $20 \mathrm{~g} / \mathrm{L}$ lidocaine was conventionally used for retrobulbar nerve blocking anesthesia and effective softening of bulbar compression.

This paper selected the upper 10:30-1:30 area for fornix-based conjunctival flaps and the rear area of corneal limbus for sclera tunnel incision with the width of $2.5 \mathrm{~mm}$, and entered the anterior chamber at $1.0 \mathrm{~mm}$ in the clear area, wherein the width of internal incision is $3.0 \mathrm{~mm}$. The tracheotomy operation for puncture auxiliary incision was made at 2.00. The sodium hyaluronate was directly injected in the anterior chamber for capsulorhexis and hydrodissection operation based on the self-made discussion needles. The apparatus fit for the phacoemulsification is Alcon Legacy 2000 with the flow of $30 \mathrm{~mL} / \mathrm{min}$ and the negative pressure of $140 \mathrm{mmHg}$. As the energy of $0-50 \%$ and time period of (1-5) minutes, emulsification lens nucleus sucked off the residual cortex relying on the automatic injection system. The foldable intraocular lens of $6.0 \mathrm{~mm}$ was planted into the bag and the carbachol was used to promote the corestenoma. The both sides were cut off alongside the sclera tunnel and the sclera was vertically scissored to the internal incision. A rectangular scleral flap was thus formed at the original incision of sclera tunnel, wherein the subvalvular trabecular meshwork and iris were cut off at the same time and the sodium hyaluronate inside of the anterior chamber was sucked out at last (4).

This paper used nylon wires of $10-0$ to interruptedly suture the scleral flaps, employed electrocoagulation to block conjunctival flaps, conducted a subconjunctival injection of antibacterial agents, ensheathe the operated eyes and made conventional postoperative treatments. This paper observed and summarized the situation of changes in 
vision, intraocular pressure and anterior chamber after the treatment. If a cataract patient fails to be treated timely and effectively, the glucose meningitis would occur, as shown in Figure 3 . Therefore, strengthening the in-depth study on the effective treatment means for cataract combined with glaucom will be of a great importance (5).

\section{RESULTS}

\section{Situation of changes in intraocular pressure}

Upon the successful completion of 48 cases of intraocular surgery, follow-up visits with the period of 3-41 months were conducted. The intraocular pressure of patients was $15.87-32.95 \mathrm{mmHg}$ before the surgery, averaged at $(23.11 \pm 2.15) \mathrm{mmHg}$. The eventual intraocular pressure of patients was $7.10-20.46 \mathrm{mmHg}$ after the surgery, averaged at $(13.64 \pm 1.58) \mathrm{mmHg}$. It can thus be seen that the intraocular pressure of patients have been effectively reduced after the surgery.

\section{Situation of changes in vision}

The international standard visual acuity chart was adopted to check the vision before and after the treatment. The vision was 0.4 before the surgery and the eventually corrected vision was $0.05-1.0$. The vision of patients has been significantly improved after the surgery, as shown in Table 1. After the treatment, the astigmatism was 0-3.00D with an average value of $0.80 \mathrm{D}$. There were 2 eyes without the astigmatism, 1 eye having irregular 3.00D astigmatism due to corneal inflammatory reaction, and the remaining 45 eyes with the astigmatism $<$ 1.50D. There were 23 eyes (47.92\%) have the astigmatism of $(0.25-0.75) \mathrm{D}$ and 22 eyes $(45.83 \%)$ have the astigmatism of (1.00-1.50) D. The averaged vision of patients has been 
significantly improved and the averaged astigmatism has been significantly reduced.

\section{Situation of anterior chamber}

After the treatment, in these 48 eyes, 6 eyes $(12.50 \%)$ were level 1 shallow anterior chamber, 1 eye $(2.08 \%)$ was level 2 , and no level 3 shallow anterior chamber. The depth of anterior chamber was returned to normal by mydriasis, high osmotic dehydration, corticosteroids and other treatments.

\section{Filtering bleb and postoperative complications}

The follow-up results showed that, there were 2 eyes $(4.17 \%)$ for non-functional filtering bleb (III type) and 46 eyes (95.83\%) for functional filtering bleb (I, II, III type). After the treatment, 3 eyes occurred for corneal edema but spontaneous recovery appeared after $7 \mathrm{~d}$. In this process, 1 eye was pupil deformation and 1 eye was shallow anterior chamber. The incidence rate of the postoperative complications was $10.42 \%$.

\section{DISCUSSION}

As a common disease in the ophthalmology clinical treatment, glaucoma patients may suffer from lens opacity at various degrees, causing serious impact on normal work and living. Currently, there mainly are 3 clinical means to treat the glaucoma combined with cataract: agents, surgery and laser therapy (6). The surgery is the most common, effective and direct therapy method applied clinically. However, numerous clinical results showed that, only applying the glaucoma surgery way to treat cannot gain an ideal therapeutic effect (7). The clinical results of Zhenyou ZHENG (8) et al showed that, when only applying the glaucoma 
surgery way to treat glaucoma combined with cataract, the vision improvement has been reduced and even worse than before the surgery.

The main advantages of phacoemulsification when applied are less trauma, effective maintenance for the depth of anterior chamber and stabilization of intraocular pressure, as well as the reduction in the incidence rate of intraoperative complications. The slighter postoperative inflammation and smaller astigmatism could create better conditions for the formation of filtering blebs. Applying the combined phacoemulsification and trabeculectomy can play a good complementary part and thus significantly improve the eventual therapeutic effect (9). It could effectively control the intraocular pressure and promote a better recovery of vision. By deepening the anterior chamber and removing the upillary block, this therapy method can promote the effective enhancement of aqueous outflow degree, effective expansion for the opening scale of anterior chamber angle and effective relief for the intraocular pressure.

The clinical results by Haijian WU have showed that, after applying the combined phacoemulsification and trabeculectomy to treat cataract combined with glaucoma, the intraocular pressure can be significantly reduce and rapidly return to a level approximating the basic intraocular pressure. Removing the inflated lens during surgery can effectively remove the block of lens and iris diaphragm and thus for better recovery of anterior chamber after the surgery and better improvement of vision. At present, there are numerous clinical studies showing that, applying the combined phacoemulsification and trabeculectomy to treat cataract combined with glaucoma could gain an ideal therapeutic effect (11).

This paper showed that, those patients of glaucoma combined with cataract 
accepting the combined phacoemulsification and trabeculectomy obtained a relatively stable intraocular pressure during surgery and returned to approximately basic intraocular pressure after the surgery. The averaged vision of patients has been significantly enhanced, whose result is close to other clinical study results. However, in the actual surgery, the protection for the corneal endothelium and anterior chamber angle by sodium hyalurantae should be fully used to prevent the rupture of posterior capsulorhexis, abjection of vitreum and congestion of the filtration road. At the same time, the lens cortex should be fully sucked out to reduce the incidence rate of postoperative complications.

Above all, applying the combined phacoemulsification and trabeculectomy for treating the cataract combined with glaucoma has a more ideal effect of intraocular pressure control. Besides, it also has advantages of slight incision, small trauma and postoperative astigmatism as well as low incidence rate of postoperative complications. Therefore, with this means, the vision can be recovered more quickly and greatly. Due to slight postoperative reactions, it could promote the quicker recovery of patients.

\section{ACKNOWLEDGEMENT}

Karamay municipal project number: JK2014-13 


\section{REFERENCES}

1. Tianlan Z. Application of phacoemulsification and intraocular lens implantation with the goniosynechialysis for angle-closure cataract combined with glaucoma. J Chin $\mathbf{J}$ Aesthetic Med 2012; 15: 315-6.

2. Jingling Z. Application for phacoemulsification and intraocular lens implantation in angle-closure cataract combined with glaucoma. J Recent Advan in Ophtha 2013; 1: $362-3$.

3. Ling W, Zhenyou Z, Jianqiang X. Analysis on clinical curative effect for the cataract extraction with intraocular lens implantation in angle-closure cataract combined with glaucoma. J Recent Advan in Ophtha 2012; 18(09): 652-653.

4. Xiaopeng L, Jianwei G, Shuang W. Clinical curative effect observation for the phacoemulsification and intraocular lens implantation in primary and angle-closure cataract combined with glaucoma. J Xinxiang Med College 2015; 11(02): 465-466.

5. Fang W, Xiao F, Jiantao W. Application of phacoemulsification and intraocular lens implantation with endoscopic cyclophotocoagulation in cataract combined with glaucoma. J Recent Advan in Ophtha 2014; 08(05): 582-583.

6. Chenggang L, Nianjun C. Clinical effect of phacoemulsification with goniosynechialysis in angle-closure cataract combined with glaucoma. J Recent Advan in Ophtha 2013; 12(02): 401-402.

7. Yanbin L. Comparison for effects of the phacoemulsification and cataract combined with glaucoma in angle-closure cataract combined with glaucoma. J Clin Rational Drug Use 2014; 17(33): 98-99. 
8. Ling W, Zhenyou Z, Jianqiang X. Analysis on clinical curative effect for the cataract extraction with intraocular lens implantation in angle-closure cataract combined with glaucoma. J Recent Advan in Ophtha 2012; 16(09): 111-112.

9. Fang W, Xiao F, Jiantao W. Application of phacoemulsification and intraocular lens implantation with endoscopic cyclophotocoagulation in cataract combined with glaucoma. J Recent Advan in Ophtha 2014; 02(05): 360-361.

10. Haijian W, Lingyan J, Zhiwei X. Comparison for effects of acute angle-closure cataract combined with glaucoma by different treatment methods. J Modern Doctor of China 2013; 08(15): 471-472.

11. Ling M. Clinical study for single-incision and double-incision cataract combined with glaucoma in the angle-closure cataract combined with glaucoma.J Modern Chin and West Med 2014; 17(33):528-529. 
Table 1: Situation for Patients' Vision before and after the Treatment [eye (\%)]

\begin{tabular}{|c|c|c|c|c|}
\hline Time & $\begin{array}{l}\text { Number } \\
\text { eye }\end{array}$ & of $\geq \mathbf{0 . 6}$ & $0.2-0.5$ & $<0.2$ \\
\hline before & 48 & 0 & $11(22.92)$ & $37(77.08)$ \\
\hline after & 48 & $31(64.58)$ & $13(27.08)$ & $4(8.33)$ \\
\hline
\end{tabular}




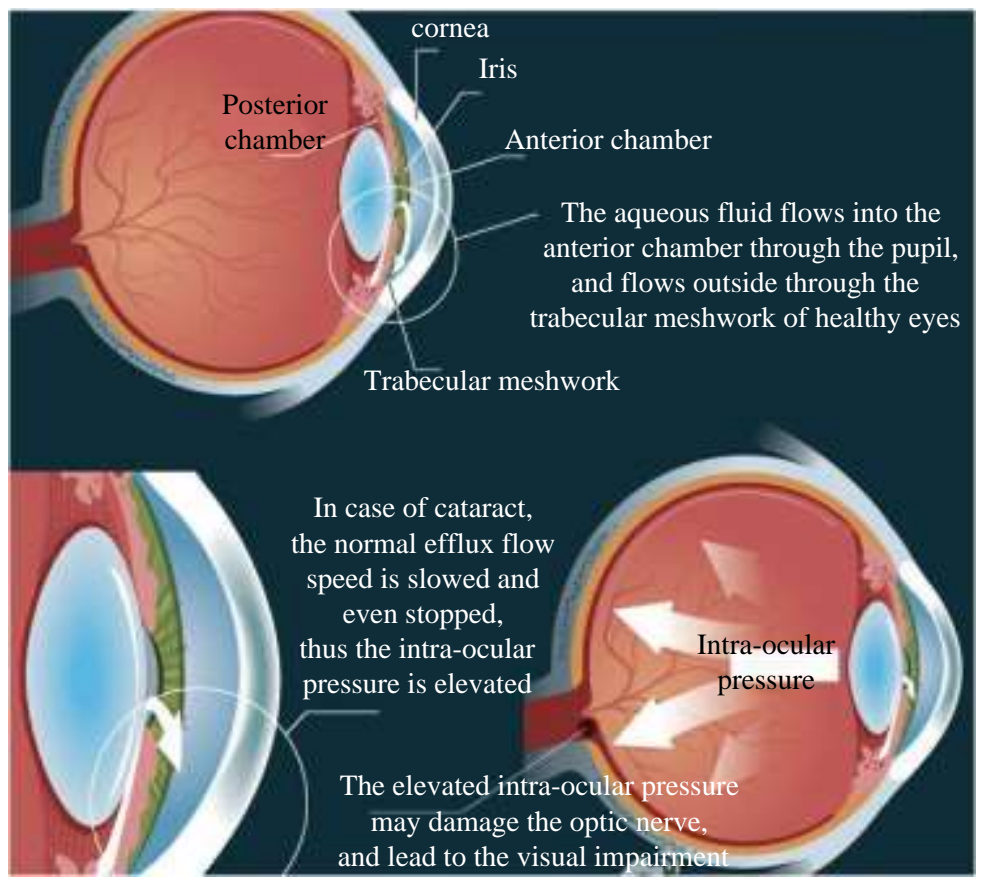

Fig. 1: Surgery of cataract combined with glaucoma.

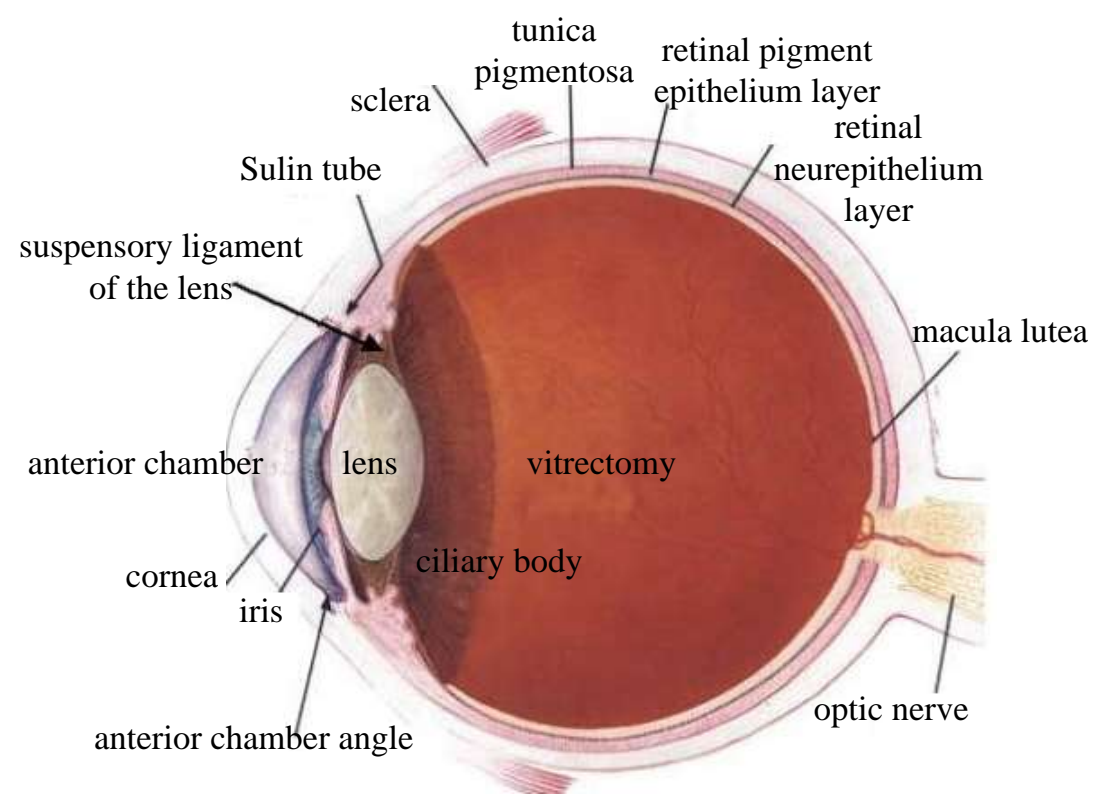

Fig. 2: Lens under a state of cataract. 


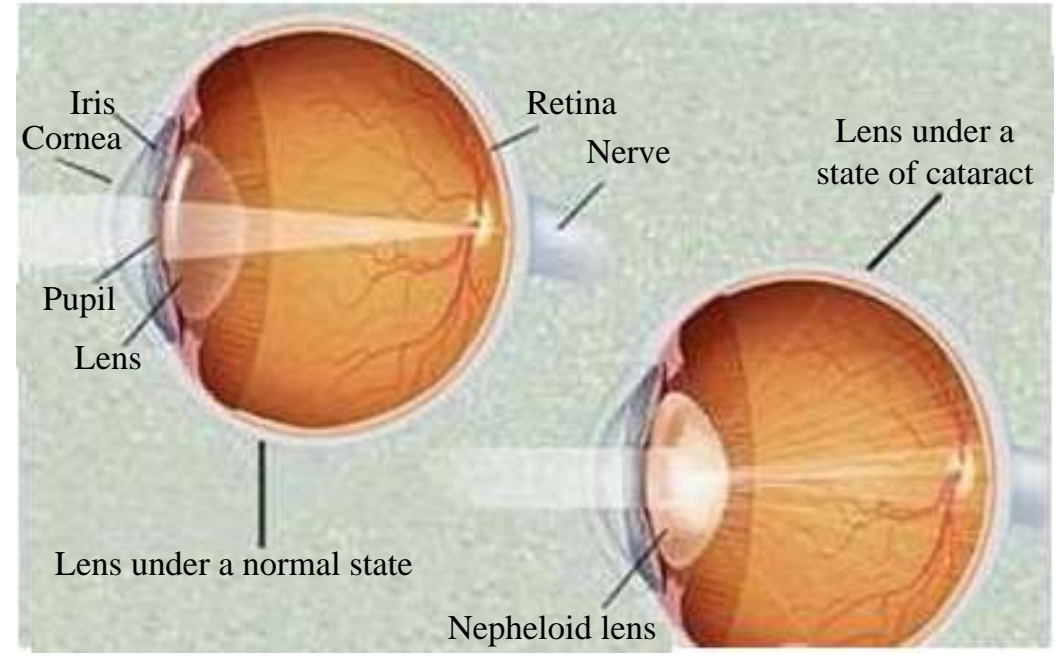

Fig. 3: Cataract leads to glucose meningitis. 